\title{
Tumor growth inhibition by synthetic and expressed siRNA targeting focal adhesion kinase
}

\author{
KAE TSUTSUMI, TATSUHIKO KASAOKA, HYI-MAN PARK, \\ HIROKO NISHIYAMA, MOTOWO NAKAJIMA ${ }^{1}$ and TOSHIYUKI HONDA \\ Tsukuba Research Institute, Novartis Pharma K.K., Ohkubo 8, Tsukuba-shi, Ibaraki 300-2611, Japan
}

Received February 13, 2008; Accepted April 3, 2008

\begin{abstract}
Focal adhesion kinase (FAK) was first identified as a viral Src substrate, and substantial experimental data have significantly correlated the elevated FAK expression in human tumor cells with an increased cell adhesion and invasion potential. However, studies investigating the role of FAK in cell proliferation have been limited. Recently, a technique known as RNA interference (RNAi) was successfully adapted to mammalian cells to decrease specifically the expression of targeted cellular genes. In this study, we investigated the role of FAK in cell proliferation, adhesion, and migration by using small interfering RNA (siRNA) technique. Firstly, we constructed a plasmid library expressing short hairpin RNAs (shRNAs) targeting FAK and selected clones substantially suppressing FAK expression in HeLa and HT1080 cells. We then studied the function of FAK in the highly invasive human prostate cancer cell line, PC $3 \mathrm{M}$, and mouse breast cancer cell line 4T1, by using selected shRNA clones (\#40 and \#42) and siRNAs chemically synthesized following the target sequences of \#40 and \#42. We demonstrated that the decrease of FAK protein expression by treatment with shRNA/siRNA targeting FAK inhibited cell adhesion on a fibronectin/laminin-coated plate, cell migration in a haptotactic migration assay, and cell proliferation in vitro. Furthermore, it suppressed tumor growth in vivo in heterotopic/orthotopic mice models. These results support our hypothesis that FAK plays a crucial role in tumor formation and growth in vivo by regulation of cell adhesion and proliferation by FAK-dependent signals.
\end{abstract}

Correspondence to: Dr Tatsuhiko Kasaoka, Tsukuba Research Institute, Novartis Pharma K.K., Ohkubo 8, Tsukuba-shi, Ibaraki 300-2611, Japan

E-mail: tatsuhiko.kasaoka@novartis.com

Present addresses: ${ }^{1} \mathrm{New}$ Business and Technology Transfer, Johnson \& Johnson K.K., 3-5-2 Nishi-kanda, Chiyoda-ku, Tokyo 101-0065, Japan

Key words: focal adhesion kinase, tumor growth, cell adhesion, RNAi library

\section{Introduction}

Malignant transformation and tumor progression are driven by complex and multi-step molecular changes in tumor cells and their microenvironment. Cellular adhesion systems, commonly altered during these molecular changes, affect tumor progression. The interactions of integrins, a class of cell adhesion receptors, and extracellular matrix (ECM) proteins transduce biochemical signals into the cell interior and induce multiple biological responses. The integrin/ECM interactions are followed by binding/activation of a number of different signaling proteins. One such pathway immediately downstream of these interactions, focal adhesion kinase (FAK) pathway, is considered to be critical in inducing cellular functional changes. Integrin signaling by means of the FAK pathway has been shown to regulate several critical cellular processes including growth, differentiation, adhesion, motility, and apoptosis $(1,2)$. FAK also plays an important role in the assembly of several signal proteins to focal adhesions via interactions with a number of cellular proteins, including Src, Grb2, phosphatidylinositol-3 kinase (PI3K), paxillin, Crk, talin, and gp130 cas (3-8). These functions of FAK are highly regulated in normal cells and appear to be perturbed in tumor cells. In studies with human tissues, FAK was found to be abundantly expressed in colon, breast, thyroid, and prostate cancers (9-12). Increasing levels of FAK expression in these cancers are reported to be correlated with the later stages of tumor progression.

It has been reported that FAK-mediated signals facilitate cell survival and are critical for efficient cell migration in response to the activation of growth factor receptors and integrin stimulation (13-16). Hungerford et al reported the blocking of FAK binding to integrin by a peptide or antibody induced the rapid cell death of fibroblasts (17). The overexpression of FAK rescued epithelial cells from apoptosis and both Y397 and K454 were critical for this effect (18). However, studies on the role of FAK related to cell proliferation have been limited even in vitro. Overexpression of FAK in $\mathrm{CHO}$ cells promoted cell cycle by increased expression of cyclin D (19). The treatment of non-small cell lung carcinoma with antisense FAK oligonucleotides resulted in decreased proliferation in the colony formation assay (20). More relevant evidence for the role of FAK in tumor cell growth, is that FAK-overexpressed HL60 cells exhibited enhanced cell growth in vitro through upregulation of cyclin D3 (21). 
Forced overexpression of FAK-related non-kinase (FRNK), a non-catalytic carboxyl terminal fragment of FAK, inhibited integrin signaling through FAK and blocked integrin-regulated events such as cell spreading, cell migration, and growth factor signaling (22-24) because FRNK acts as a dominant negative inhibitor of FAK resulting in constitutive reduction of tyrosine phosphorylation of FAK and blocking of the formation of focal adhesions $(22,25)$.

In order to investigate the role of a gene of interest, a number of techniques have been used to suppress its expression and functions, including antisense oligonucleotides and dominant negative forms of the target gene. The recently developed small interfering RNA (RNAi) technique seems to be more potent and efficient than antisense oligonucleotides in reducing target gene expression in vitro, and has also emerged as a powerful tool for silencing gene expression even in animals $(26,27)$. The mediators of RNAi have been observed to be 21- to 22-nt siRNAs generated by RNase III cleavage from longer dsRNA, the delivery of which has been shown to knock down the expression of specific proteins in insect cells $(28,29)$. It has also been shown that the delivery of 21-nt siRNA specifically suppressed expression of endogenous and heterologous genes in both cultured mammalian cells in vitro and adult mice in vivo (30-33). Furthermore, several recent reports have demonstrated the usefulness of RNA Pol III promoter for the expression of siRNA molecules (34-40).

In this study, we employed both chemically synthesized siRNA oligonucleotides and short hairpin RNA (shRNA)expressing plasmids targeting FAK mRNA in order to validate the role of FAK in tumor progression, especially in tumor proliferation. To screen the effective siRNA targeting sequence of human FAK, we constructed a plasmid library expressing shRNAs targeting FAK and then isolated the clones efficiently suppressing endogenous FAK. Chemically synthesized siRNA oligonucleotides following the target sequence of the shRNA also suppressed the expression of FAK protein to the same level as did shRNA. Decreased FAK protein expression by either shRNA- or siRNA-treatment potently inhibited cell migration and adhesion on fibronectin/laminin coated plates. In addition, we demonstrated that siRNA targeting FAK inhibited cell proliferation in vitro and suppressed tumor growth in mice. Collectively, these results strongly support the hypothesis that FAK regulates tumor growth in vivo by upregulation of cell adhesion and proliferation through FAKdependent signals.

\section{Materials and methods}

Cell culture. Human prostate cancer cell line PC3M, was maintained in RPMI-1640 medium (Sigma, St. Louis, MO, USA) and mouse breast cancer cell line 4T1, was maintained in DMEM (Invitrogen, Carlsbad, CA, USA), supplemented with $10 \%$ fetal bovine serum (FBS; JRH Biosciences, Lenexa, $\mathrm{KS}, \mathrm{USA}$ ) and Antibiotic-Antimycotic (Invitrogen) at $37^{\circ} \mathrm{C}$ in an atmosphere of $5 \% \mathrm{CO}_{2}$ in air.

Preparation and screening of the shRNA plasmid library. Construction of a plasmid library expressing shRNAs and the determination of target sequences to suppress endogenous human FAK was performed by GenoFunction Inc. (Ibaraki, Japan). Briefly, shRNA expressing vector was constructed by modifying pUC19 to express shRNA under the control of human U6 promoter. To generate a shRNA plasmid library, human FAK cDNA (GenBank accession no. L13616) cloned in pGEMT vector (Promega, Madison, WI, USA) was amplified by PCR with SP6 and T7 promoter primers, digested with DNase and separated into 25-32 base pair fragments by agarose electrophoresis. The sense and antisense oligonucleotides of each fragment were constructed as one-opened single-stranded fragments by ligating an interstitial loop sequence, GAAAA, between the strands. These oligonucleotides were amplified by PCR with addition of a human U6 promoter in the 5 '-end and a terminator in the 3 '-end. Resultant DNA fragments expressing shRNA were inserted into the EcoRI and HindIII site of pUC19 vectors.

To assess the quality of this library, 32 clones were randomly selected from $1 \times 10^{5}$ clones of the library and sequenced. The sequencing results showed that 20 clones coded the FAK sequence and covered $13 \%$ of the FAK cDNA without overlapping each other. In the first screening, a total of 168 clones were assessed. Each plasmid was transfected into HT1080 cells in 96-well plates and the potency of each clone in silencing the endogenous FAK mRNA was assessed by real-time RT-PCR $48 \mathrm{~h}$ after transfection. Twenty-six clones were selected in order of the RNA interference activity and subjected to the second screening in 12-well plates using HT1080 cells. Sixteen clones silencing target mRNA by more than $50 \%$ were further confirmed to be effective in HeLa cells (Fig. 1). We picked up 4 clones \#40, $\# 42$, \#43 and \#64 from the diversity of their coding region in FAK cDNA for further study using a human cancer cell line. For study using a mouse cancer cell line, we chose clones \#40, \#42, \#64 and \#66 for their high homology with mouse FAK (no mismatch in \#40 and one mismatch in the other three clones) as well as the diversity of their coding region in FAK cDNA.

Preparation of siRNA oligonucleotides targeting FAK. From the results of the characterization of selected clones from the shRNA plasmid library, sense and antisense RNAs targeting FAK and negative control (scrambled siRNA) were chemically synthesized by Japan Bio Service (Saitama, Japan). The targeting sense strands sequences for FAK were from position 1109 to 1134 (\#40) and 1990 to 2017 (\#42) relative to the start codon. Scrambled RNA was 5'-CAGUCGCGUUUG CGACUGGTT-3'. These RNAs were dissolved in diethylpyrocarbonate water as $100 \mu \mathrm{M}$ solutions. Double-stranded siRNAs were generated by mixing the corresponding pair of sense and antisense RNA oligonucleotides at $20 \mu \mathrm{M}$ concentration in annealing buffer [30 mM HEPES-KOH ( $\mathrm{pH} 7.4)$, $100 \mathrm{mM}$ potassium acetate, and $2 \mathrm{mM}$ magnesium acetate]. The reaction mixture was heated at $90^{\circ} \mathrm{C}$ for $1 \mathrm{~min}$, allowed to reach room temperature over $1 \mathrm{~h}$, and then aliquoted and stored at $-30^{\circ} \mathrm{C}$.

Transfection of shRNA plasmids and siRNA oligonucleotides. For the transfection of shRNA plasmids, PC $3 \mathrm{M}$ cells $\left(5 \times 10^{4}\right.$ cells/well) were seeded into 12 -well plates (Corning Inc., Corning, NY, USA) in $1 \mathrm{ml}$ of medium one day prior to 


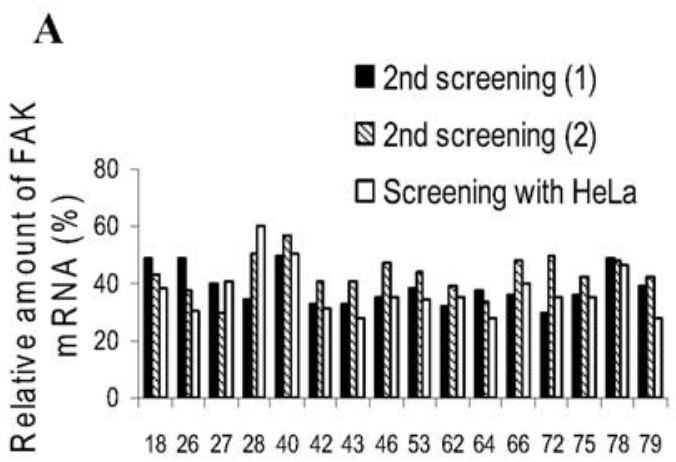

Clone number

B

\begin{tabular}{l} 
Clone no. $\begin{array}{c}\text { Target sequence } \\
\text { (sequence from the promoter) }\end{array}$ \\
\hline 18 TACTGGTATGGAACGTTCTCCTGGTGC \\
26 GCCTGTGGGTAACCAGATCCTGCAGCT \\
27 GACCGGGTTATCCCAGTCCGAGGTCC \\
28 GGCAAGTGTTGCCAACCCATCTGAT \\
40 TGCCATCAATACCAAAGTTGGCCAAC \\
42 TAAGTTCAGTAAACCTGGGCCGCCTGCT \\
43 GAAGCTATTGAACTCTGACCTGGG \\
46 AGATATGCAAGAGCTGTACTAAGC \\
53 TGCGGGGCAATGCACTAGAAAAGA \\
62 CAGCACCCACCGAGAGATTGAGATGGCACA \\
64 TAAGCCAACTTTGAATTCTTCTATC \\
66 TGTGGGCTGGGATAAAATCCTTCGCT \\
72 GAGGTCCAGCGAAGGATTTATCCC \\
75 GGCCCAGCAGTATGTCATGACCAGC \\
78 GGAGTGGAAATATGAATTGAGAATTC \\
79 TGGCTGGCAGCATCTATCCAGGTCAGG \\
\hline
\end{tabular}

Figure 1. Characterization of 16 clones selected from the shRNA library against FAK. (A) Inhibition activities of clones from the shRNA library against FAK mRNA expression in mammalian cells assessed by real-time RT-PCR. Each clone was transfected into the HT1080 (2nd screening 1 and 2) or HeLa cells. Total RNA was extracted from the cells $48 \mathrm{~h}$ after the transfection and subjected to real-time RT-PCR. The results were shown as the relative amount of FAK mRNA to the control which was transfected with empty pUC19 vector. (B) The target sequence of each clone.

transfection. Transfection of shRNA plasmid was performed using Lipofectamine 2000 Reagent (Invitrogen). Plasmid $(2 \mu \mathrm{g})$ was transfected using $2 \mu 1$ of Lipofectamine 2000 Reagent in Opti-Mem I medium (Invitrogen). Cells were washed with PBS $4 \mathrm{~h}$ after transfection and further incubated with the culture medium. The following day, transfected cells were harvested with Trypsin-EDTA (Sigma) and split into new dishes for the studies. Transfection conditions for 4T1 cells were basically the same as described above except that the seeding cell density was at $2.5 \times 10^{5}$ cells/well in $1 \mathrm{ml}$ of medium and the volume of Lipofectamine 2000 Reagent used was $8 \mu$ l. Plasmid coding enhanced green fluorescence protein (EGFP) driven by a CMV promoter (Clontech, Palo Alto, CA, USA) and/or pUC shRNA against luciferase (shLuc) targeting sense strand sequence (5'-TCAAAGTGCG CTGCTGGTGCCAACCCTAT-3') were used for control.
For the transfection of siRNA oligonucleotides, PC3M and $4 \mathrm{~T} 1$ cells suspended in $3 \mathrm{ml}$ of medium containing $10 \%$ FBS were seeded into each well of a 6-well plate (Corning Inc.) and cultured to give $50 \%$ confluence one day prior to the transfection. Cells were washed twice with each medium without FBS, and then the transfection of siRNAs was performed using Oligofectamine Reagent (Invitrogen) in the final siRNA concentration of $240 \mathrm{nM}$ in $1 \mathrm{ml}$ of antibioticantimycotic free medium without FBS. Cells were then incubated for $4 \mathrm{~h}$ and cultured further in conditioned medium supplemented with $10 \%$ FBS. After incubation for $24 \mathrm{~h}$, cells were washed, and the culture medium was replaced with fresh medium supplemented with $10 \%$ FBS. The cell passages were performed as described above.

Western blot analysis. Transfectant cells were harvested and lysed in mRIPA buffer [50 mM Tris- $\mathrm{HCl}(\mathrm{pH} 7.4), 1 \%$ (v/v) $\mathrm{NP}-40,0.25 \%$ (w/v) sodium deoxycholate, $150 \mathrm{mM} \mathrm{NaCl}$, and $1 \mathrm{mM}$ EDTA] containing phosphatase inhibitor and protease inhibitors $\left(1 \mathrm{mM} \mathrm{Na} \mathrm{VO}_{4}, 1 \mathrm{mM} \mathrm{NaF}, 10 \mu \mathrm{g} / \mathrm{ml}\right.$ pepstatin, and $30 \mu \mathrm{M}$ Cal I) after washing twice with PBS. Lysates were then centrifuged for $10 \mathrm{~min}$ at $4^{\circ} \mathrm{C}$ to remove debris, the protein concentration was measured using a BCA protein assay kit (Pierce, Rockford, IL, USA) with bovine serum albumin as a standard, and the solutions were stored at $-30^{\circ} \mathrm{C}$ until immunoblotting was performed.

Cell lysate $(10 \mu \mathrm{g})$ was separated on $10 \%$ SDS-polyacrylamide gels and electrotransferred to $0.2 \mu \mathrm{m}$ Immobilon-PSQ PVDF membranes (Millipore, Bedford, MA, USA) using the wet transblot method in transfer buffer [50 mM Tris- $\mathrm{HCl}$ ( $\mathrm{pH} 7.6), 40 \mathrm{mM}$ glycine, $1 \mathrm{mM}$ SDS, and $20 \%$ (v/v) methanol]. After blocking with TBS-T [50 mM Tris-HCl (pH 7.6), $150 \mathrm{mM} \mathrm{NaCl}$, and $0.1 \%$ (v/v) Tween-20] containing 5\% skim milk powder (Becton Dickinson, Sparks, MD, USA) for $30 \mathrm{~min}$ at room temperature, membranes were incubated for $2 \mathrm{~h}$ at room temperature or overnight at $4{ }^{\circ} \mathrm{C}$ with the first antibody. The following were used as the first antibodies: anti-FAK mouse monoclonal antibody (clone 4.47; Upstate Biotechnology, Lake Placid, NY, USA), antiFAK rabbit polyclonal antibody (no. 06-543; Cell Signaling Technology), anti-extracellular-regulated kinase (ERK) rabbit polyclonal antibody (no. 9102; Cell Signaling Technology), anti-phospho ERK rabbit polyclonal antibody (no. 9101L; Cell Signaling Technology), anti-GAPDH rabbit polyclonal antibody (Trevigen, Gaithersburg, MD, USA), anti-ß-actin mouse monoclonal antibody (no. 9101L; Abcom, Cambridge, UK). The membranes were washed 3 times with TBS-T and then incubated for $1 \mathrm{~h}$ at room temperature with anti-mouse or rabbit $\mathrm{IgG} /$ horseradish peroxidase antibody (Amersham Biosciences, Rochester, NY, USA) in TBS-T containing 5\% skim-milk powder as the secondary antibody for FAK. After washing three times with TBS-T, the immunoreactive bands were visualized using enhanced chemiluminescence detection reagents (Amersham Biosciences). Images were captured by a luminescent image analyzer, LAS-3000 (Fuji Film, Tokyo, Japan), and the signals were quantified using Image Gauge software program ver. 4.0 (Fuji Film).

Cell adhesion assay. Two days after transfection, PC3M and 4T1 transfectant cells were harvested with Trypsin-EDTA 
and seeded at a concentration of $2 \times 10^{4}$ or $5 \times 10^{4}$ cells/well in each medium, respectively, on a 96-well plate (Corning Inc.) coated with $0.5 \mu \mathrm{g} /$ well fibronectin or $0.25 \mu \mathrm{g} /$ well laminin. After $1 \mathrm{~h}$ of incubation at $37^{\circ} \mathrm{C}$, cells were washed with PBS and non-adherent cells were removed by aspiration. Adherent cells were stained with $0.5 \%$ crystal violet diluted in $20 \%$ methanol and rinsed with distilled water. The dye in the stained cells was solubilized by adding $1 \%$ SDS and the absorbance was measured at a wavelength of $595 \mathrm{~nm}$ with a microplate reader (ThermoMax; Molecular Devices, Sunnyvale, CA, USA). The difference in absorption between FAK siRNA-treated and control (scrambled siRNA in PC3M and shLuc in 4T1) cells was compared for statistical significance using the unpaired, two-tailed t-test with Bonferroni correction.

Haptotactic migration assay. Haptotactic cell migration assay was carried out using Transwell (12-mm diameter with $8-\mu \mathrm{m}$ pores) cell culture chambers (Corning Inc.). The lower surface of the filter was pre-coated with $1 \mu \mathrm{g}$ of fibronectin. PC3M and 4T1 transfectant cells $\left(1 \times 10^{5}\right.$ cells $)$ cultured for 2 days after transfection were added to the upper compartment and incubated for $3 \mathrm{~h}$ at $37^{\circ} \mathrm{C}$. The filters were fixed with $30 \%$ methanol, stained with $0.5 \%$ crystal violet in $20 \%$ methanol, and the cells that remained on the upper side of the filter were removed with cotton tip applicators. The filters containing the stained cells that had invaded to their lower sides were removed from the Transwell chambers and individually transferred to separate wells in a 96-well culture plate. The crystal violet dye retained on the filters was solubilized by adding $1 \%$ SDS and colorimetrically assessed by measuring its absorbance at a wavelength of $595 \mathrm{~nm}$. The difference in absorption between the cells treated with FAK siRNAs and the control transfected cells was compared for statistical significance using the unpaired, two-tailed t-test with Bonferroni correction. The controls were transfected with scrambled siRNA in PC3M cells or with shLuc in 4T1 cells.

Cell proliferation assay. For normal plate assay, PC3M and $4 \mathrm{~T} 1$ cells were seeded at a concentration of $2.5 \times 10^{3}$ or $2 \times 10^{3}$ cells/well, respectively, on a 96-well plate one day before transfection. The transfection with siRNAs in PC3M cells and shRNAs in 4T1 cells were performed under the same conditions as described above. After a 4-h incubation, culture media were changed to phenol red-free RPMI-1640 medium (Invitrogen) supplemented with 5\% FBS, and further cultured. For laminin-coated plate assay, PC3M and 4T1 cells cultured on 6-well plates after transfection, were seeded at a concentration of $2.5 \times 10^{3}$ or $2 \times 10^{3}$ cells/well in each medium on a 96-well plate coated with 0.25 or $0.0625 \mu \mathrm{g} /$ well laminin, respectively. Cell proliferation at different time points was examined by MTT method. In brief, MTT [3-(4, 5-dimethylthiazol-2-yl)-2, 5-diphenyltetrazolium bromide] was added to each well $(1 \mathrm{mg} / \mathrm{ml})$ and after $4 \mathrm{~h}$ of incubation at $37^{\circ} \mathrm{C}, 10 \%$ $(\mathrm{w} / \mathrm{v}) \mathrm{SDS}$ in $0.01 \mathrm{~N} \mathrm{HCl}$ solution was applied to the well to dissolve the formazan crystal derived from MTT. The absorbance of each well was measured at a wavelength of $595 \mathrm{~nm}$. The difference in absorption between the cells treated with FAK siRNAs and the control cells treated with scrambled siRNA in PC3M or with shLuc in 4T1 was compared for statistical significance using the unpaired, twotailed t-test with Bonferroni correction.

In vivo tumor inhibition. BALB/cAJcl-nu/nu mice (7-10 week old males) and BALB/cAJcl mice (7-10 week old females) were purchased from CLEA Japan for the study using PC3M and 4T1 cells, respectively. Mice received food and water ad libitum and were maintained in a controlled environment (12 h light/dark cycle at $\left.22-25^{\circ} \mathrm{C}\right)$. PC $3 \mathrm{M}$ and $4 \mathrm{~T} 1$ cells cultured in $10-\mathrm{cm}$ dishes were transfected with siRNA oligonucleotides or shRNA plasmids under the same conditions as described above, respectively. One day after the transfection, cells were detached from the dishes with Trypsin-EDTA. After twice washing with PBS, more than $95 \%$ cell viability was confirmed by trypan blue staining. PC $3 \mathrm{M}$ cells were injected s.c. to the abdomen of nude mice at a cell density of $3 \times 10^{6}$ cells $/ 50 \mu 1 /$ animal. $4 \mathrm{~T} 1$ cells were injected into the mammary fat pad of mice at a cell density of $1 \times 10^{6}$ cells/ $50 \mu 1$ /animal. After tumors became palpable, tumor diameters of long length and short length were measured with a caliper every 2 or 3 days and the volume of each tumor was calculated as long length $x$ (short length) $2 / 2$. The difference in tumor volume between the FAK siRNA-treated cells and the control transfected with scrambled siRNA in PC3M or with shLuc in 4T1 was compared for statistic significance using Mann-Whitney U test. All animal studies were carried out under protocols approved by Novartis Tsukuba Research Institute Animal Welfare Committee.

\section{Results}

Characterization of selected shRNA clones against FAK. We first examined the suppression of FAK protein levels by selected shRNA clones targeting FAK. Human prostate cancer PC $3 \mathrm{M}$ cells, and mouse breast cancer 4T1 cells, were transfected with shRNAs \#40, \#42, \#43 and \#64 and shRNAs \#40, \#42, \#64 and \#66, respectively. There was one mismatch nucleotide sequence against mouse FAK at the indicated position in each of shRNAs \#42, \#64 and \#66, while the sequence of shRNA \#40 was completely matched between human and mouse FAK (Fig. 2B). After transfection, cells were harvested at the indicated time and the expression levels of FAK protein were analyzed by Western blotting. In PC3M cells, all shRNA clones showed up to $90 \%$ reduction in the FAK protein expression level at 2 days after transfection compared with controls (plasmid coding EGFP or shLuc). The effect of shRNA was persistent up to day 4 , and the levels of FAK protein started to recover at day 5 (Fig. 2A). In 4T1 cells, endogenous FAK protein expression was also attenuated by the transfection of shRNA \#40 and \#42; however, their suppression degrees were not as dominant as those observed in PC3M cells (Fig. 2B). The effects of shRNA \#40 and \#42 were most prominent at day 2 and gradually decreased to very low levels by day 5 post-transfection. The other two clones had no effect on the suppression of FAK protein expression in 4T1 cells. Therefore, for further study, we selected shRNA \#40 and \#42, which were effective in both PC3M and 4T1 cells.

It has been reported that FAK is a positive regulator of EGF-stimulated JNK and ERK signaling in A549 and 293T 
A

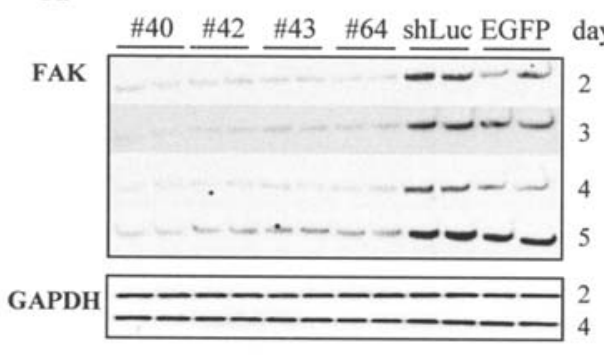

C

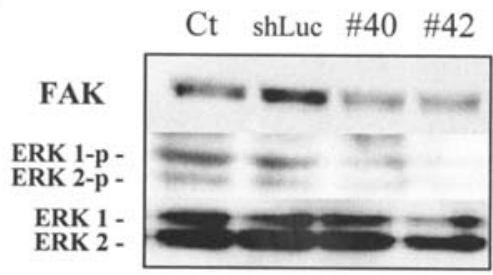

B
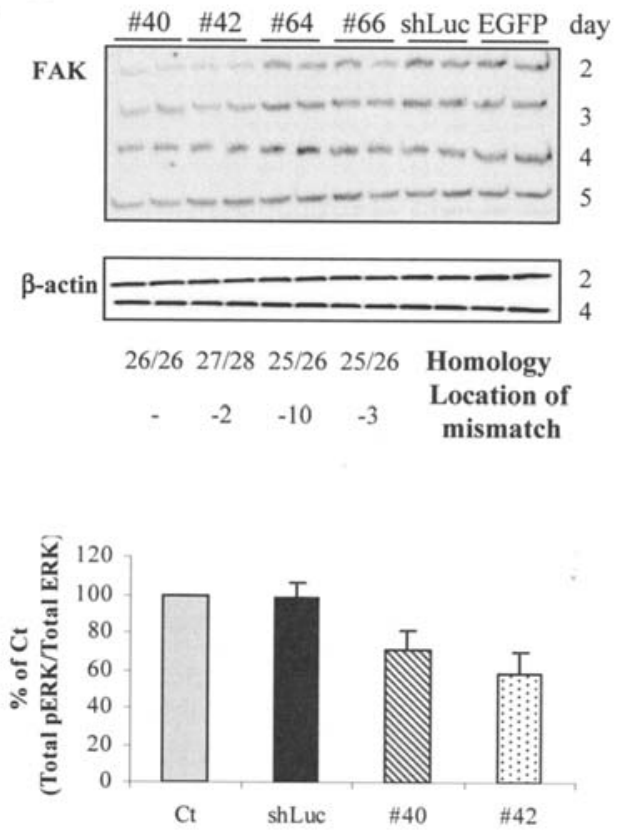

Figure 2. Inhibition of FAK protein expression and its signal transduction by the transfection of shRNA against FAK. Plasmid coding shRNA selected for human FAK, clones \#40,\#42, \#43 and \#64 or \#40, \#42, \#64 and \#66, were transfected to PC3M cells (A), or 4T1 cells (B), respectively. Plasmid expressing shLuc or EGFP was used as a control. One day after transfection, cells were trypsinized and split into new dishes for each time point of the harvest. The cells were lysed at the indicated days and subjected to Western blot analysis using anti-FAK antibody or anti-GAPDH/B-actin antibody to insure equal loading. Each treatment was duplicated in each experiment. Experiments were repeated twice with similar results. 'Homology', as noted below the panel in B refers to the homology of the sequence of each clone to the mouse sequence (the number of the same nucleotides with mouse / the number of nucleotides of shRNA). 'Location of mismatch' refers to the position of each mismatched nucleotide between human and mouse from the 3'-end of the shRNA sequence. (C) Plasmid coding shRNA selected for human FAK, clones \#40, \#42 and shLuc, were transfected to 4T1 cells cultured on a 6-well plate. After a two-day incubation, cells were lysed and subjected to Western blot analysis using anti-FAK, ERK and phosphorylated ERK antibodies.

cells (41). To confirm that the suppression of FAK expression by RNAi inhibits the signal transduction of FAK, phosphorylation of ERK was analyzed by Western blotting. In 4T1 cells, phosphorylation of ERK was attenuated by the transfection of shRNA \#40 and \#42, indicating that the suppression of FAK by shRNA impaired the downstream signal transduction of FAK (Fig. 2C).

Suppression of FAK protein expression by siRNA. In PC3M cells but not in $4 \mathrm{~T} 1$ cells, slight cell damage by the transfection of shRNA and control plasmids were observed under a microscope. To avoid cell damage, siRNA oligonucleotides were used next as small size siRNA oligonucleotides were considered to be less toxic on the cells than shRNA plasmids. At first, we optimized conditions for transfection of siRNA into PC3M cells using Cy3-labeled luciferase GL2 siRNA duplex (Dharmacon Research, Inc., Lafayette, CO, USA). The efficiency of transfection was assessed by counting the fluorogenic cells on a fluorescence microscope day 2 posttransfection. Under the optimized conditions described in Materials and methods, almost $100 \%$ of PC3M cells were positive for Cy-3 and no cell damage was observed (data not shown).

Two kinds of siRNA oligonucleotides, \#40 and \#42 were then synthesized from the corresponding sequences of shRNA plasmid \#40 and \#42, and transfected to PC3M cells under optimal conditions and to evaluate potency in inhibiting FAK protein expression. Transfected cells were harvested at each time point of 1,2, 3 and 4 days after transfection, and the expression levels of FAK protein were analyzed by Western blotting using anti-FAK antibody. As shown in Fig. 3, a significant suppression of FAK protein expression by siRNA \#42 was observed at day 1 and lasted until 4 days after transfection. SiRNA \#40 also suppressed the expression of FAK protein throughout the observation period but to a lesser extent. Maximal inhibition (50 and $80 \%$ reduction by siRNA $\# 40$ and \#42, respectively, compared with scrambled siRNA) was observed at day 3 or 4 without cell damage. When siRNA was applied to 4T1 cells under optimal conditions determined by the same method as for PC $3 \mathrm{M}$, inhibition of FAK protein expression was hardly observed regardless of the high efficiency of the introduction of oligonucleotides (data not shown). Therefore, we decided to use the chemically synthesized siRNAs in PC3M studies and shRNAs in 4T1 studies as a tool for the suppression of FAK protein expression.

Inhibition of cell adhesion by FAK siRNA and shRNA. In many cells, apoptosis following loss of adhesion may be influenced by factors that modulate initial cell adhesion to ECM proteins. Cell adhesion via the ECM-integrins is the first step in the integration of intracellular signaling through FAK leading to the initiation of cell invasion and proliferation. To determine the role of FAK in integrin-associated cell adhesion, we examined the effect of decreased levels of FAK by siRNA/shRNA on cell adhesion to the matrix protein fibronectin and laminin. The results are shown in Fig. 4. The 
A

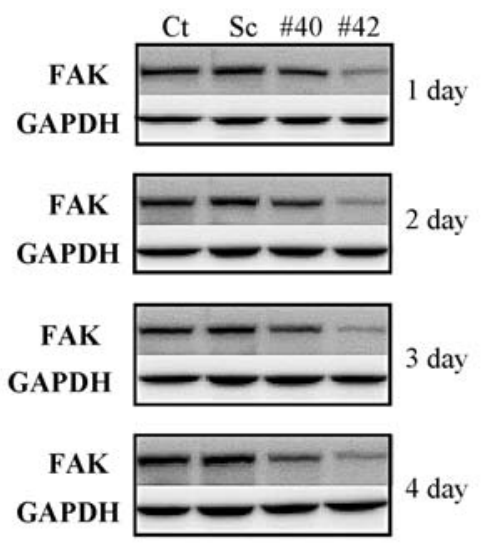

B

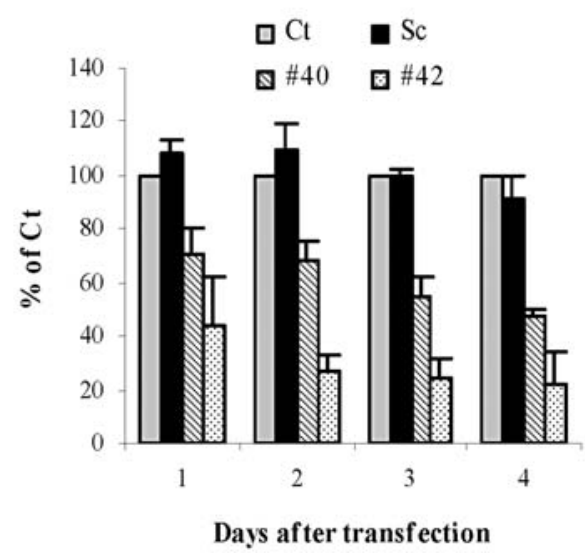

Figure 3. Suppression of FAK protein expression by siRNA in PC3M cells. (A) Overnight culture of PC3M cells seeded on a 6-well plate were transfected with scrambled siRNA or siRNA targeting FAK (\#40 and \#42), which were synthesized from the sequence of clones \#40 and \#42 shRNA. Scrambled siRNA ( $\mathrm{Sc}$ ) was used as control. One day after transfection, cells were trypsinized and split into new dishes for each time point of the harvest. The cells were lysed at the indicated days and subjected to Western blot analysis using anti-FAK antibody or anti-GAPDH antibody to ensure equal loading. (B) Image of (A) was quantified by Image Gauge ver. 4.0 software and expressed as the ratio of the FAK band intensity of the treatment with siRNA targeting FAK (\#40 or \#42) to that of the control treatment with scrambled siRNA at each time point after normalizing by GAPDH.

transfection of siRNA/shRNA \#40 and \#42 inhibited adhesion to fibronectin by 40 and $33 \%$ in PC $3 \mathrm{M}$ and 28 and $57 \%$ in $4 \mathrm{~T} 1$ cells, respectively, compared with controls. Similarly, there was a clear reduction of adhesion to laminin by siRNA/shRNA of \#40 and \#42 by 18 and 33\% in PC3M and 23 and $40 \%$ in $4 \mathrm{~T} 1$ cells, respectively.

Inhibition of cell migration by FAK siRNA and shRNA. Next, to determine the effect of FAK on haptotactic cell migration, we measured the migration ability of cells transfected with siRNAs/shRNAs targeting FAK by using modified Boyden chambers coated with fibronectin as an attractant. The results are shown in Fig. 5. The transfection of siRNAs of \#40 and \#42 inhibited migration of PC3M cells by 30 and $25 \%$, respectively, compared with scrambled siRNA control. The transfection of shRNAs of \#40 and \#42 inhibited migration of $4 \mathrm{~T} 1$ cells by 7 and $18 \%$ compared with shLuc control cells, respectively. Clear inhibition of cell migration by FAK suppression was observed in PC3M cells, however, only a slight inhibition of cell migration was observed in 4T1 cells.

Inhibition of cell proliferation by FAK siRNA and shRNA in vitro. We next examined the effect of siRNA/shRNA transfection on cell proliferation. As shown in Fig. 6A and B, FAK suppression by siRNA/shRNA retarded growth of both PC3M and 4T1 cells grown on the normal plate 3 days after transfection as compared with control transfected cells. Growth inhibition of PC3M cells by siRNA \#40 and \#42 was 28 and $18 \%$, respectively, as compared with scrambled siRNA transfected cells. Growth inhibition of 4T1 cells by shRNA $\# 40$ and \#42 was 22 and 8\%, respectively, as compared with shLuc transfectants. When the cells were cultured on laminincoated plates, the effects of FAK suppression on cell growth were more clearly observable. Maximal growth inhibition of PC 3M cells by siRNA \#40 and \#42 was 36 and $21 \%$, respectively, as compared with scrambled siRNA transfected cells. Maximal growth inhibition of 4T1 cells by shRNA \#40 and \#42 were 34 and 41\%, respectively, as compared with shLuc transfectants.

Suppression of tumor formation by FAK siRNA and shRNA treatment in mice. We examined finally whether suppression of FAK by siRNAs/shRNAs inhibits tumor growth in vivo. Nude mice injected with PC3M cells transfected with scrambled siRNA developed tumors with approximately $400-\mathrm{mm}^{3}$ volume at day 16 , whereas mice injected with PC3M cells transfected with siRNAs targeting FAK showed only minimal tumor growth (Fig. 7A). In addition, no tumor was formed in some of the mice injected with PC3M cells transfected with siRNA \#40. Day 16 post-injection siRNA FAK \#40 and \#42 showed growth inhibition by 83 and $54 \%$, respectively, as compared with scrambled siRNA. Similarly, injection of 4T1 cells transfected with shLuc control vectors produced tumors in mice within 12 days, whereas 4T1 cells transfected with shRNAs targeting FAK showed slow tumor growth (Fig. 7B). Day 12 post-injection shRNA FAK \#40 and \#42 showed growth inhibition by 30 and $32 \%$, respectively, compared with shLuc. In every study, FAK protein expression levels in the injected cells were analyzed by Western blotting when they were injected and more than $50 \%$ suppression by siRNA/ shRNA was confirmed (Fig. 7C). From the graph of logarithmic regression trend as shown in the inset, it was clearly demonstrated that PC3M tumor growth rate in vivo was effectively inhibited by siRNA until 9 days after cell injection, but not later. The growth rate of 4T1 cells was suppressed by shRNA up to 8 days after cell injection. These results indicate that the effective efficacy of siRNA/shRNA lasted more than one week as gradually diluted by cell division and degradation by RNase.

\section{Discussion}

To evaluate protein functions, researchers for years, have focused on effective tools to specifically down-regulate gene expression, such as antisense oligonucleotide strategy. However, there has been only limited success due to the lack of specificity and potency of this method (42). Doublestranded RNA is more resistant to nuclease degradation as 
A
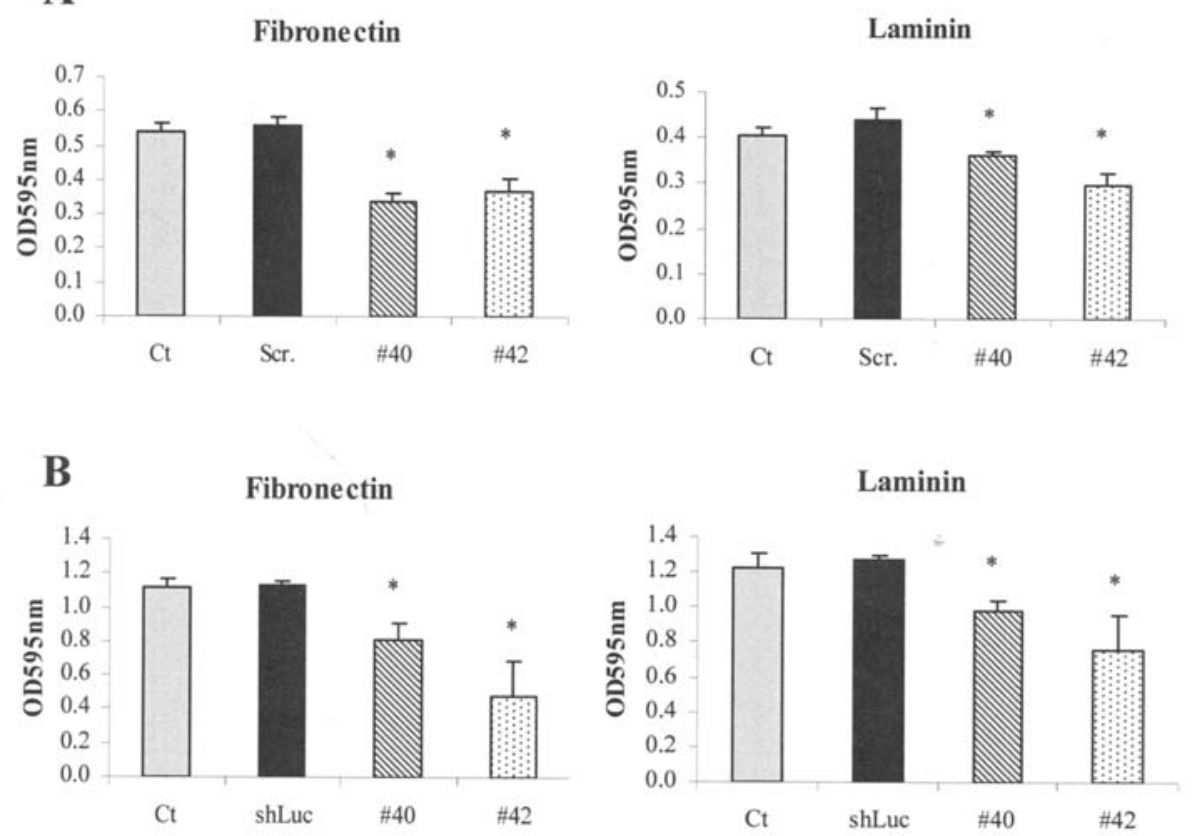

Figure 4. Adhesive properties of control (mock transfected) and transfectants of siRNA or shRNA plasmid targeting FAK. Two days after transfection with FAK or scrambled siRNA (Scr) in PC3M (A) and luciferase (shLuc) or FAK shRNA plasmid in 4T1 (B), cells were harvested and seeded on a 96-well plate coated with fibronectin or laminin. After $1 \mathrm{~h}$ of incubation at $37^{\circ} \mathrm{C}$ followed by washing with PBS, adherent cells were measured after staining with crystal violet. Results are expressed as means $\pm \mathrm{SE}$ of the average of three independent experiments $(n=3)$. ${ }^{*} \mathrm{P}<0.05$ compared with scrambled siRNA or shLuc plasmid transfectants in (A) or (B), respectively (unpaired, two-tailed t-test with Bonferroni correction).
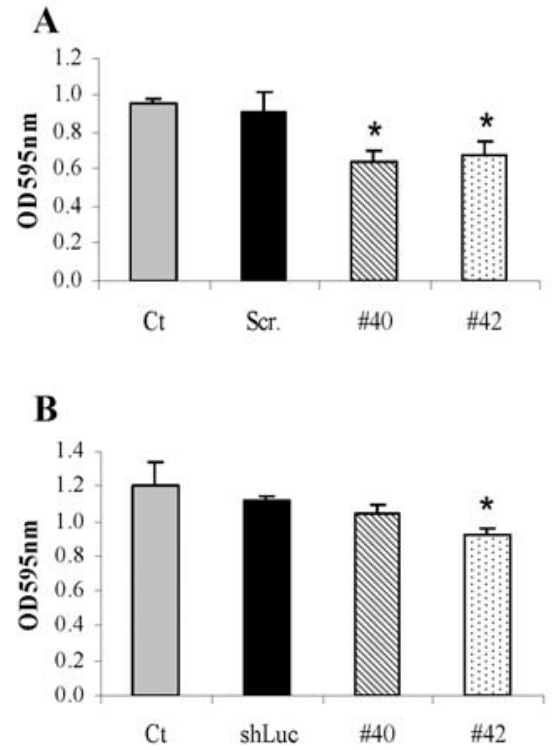

Figure 5. Inhibition of cell migration by treatment with siRNA or shRNA plasmid targeting FAK. Two days after transfection with FAK or scrambled siRNA (Scr) in PC3M (A) and luciferase (shLuc) or FAK shRNA plasmid in 4T1 (B), cells were harvested and seeded into a Transwell chamber in triplicate. After $3 \mathrm{~h}$ of incubation, the migrating cells were detected by crystal violet staining method. Results are expressed as means \pm SD of triplicate experiments. These experiments were repeated twice with similar results. ${ }^{*} \mathrm{P}<0.05$ compared with scrambled siRNA or shLuc plasmid in (A) or (B), respectively (unpaired, two-tailed t-test with Bonferroni correction).

compared with antisense oligonucleotides, so that longer lasting suppression could be expected with siRNA than with the antisense approaches. The recent progress of studies using synthetic siRNA molecules and expression systems have demonstrated a successful reduction in targeted protein expression both in vitro and in vivo. In this technology siRNA target sequence is critical for efficient suppression of the target gene, so that several algorithms to find the effective target sequence have been explored, some of which are commercially available.

Here, we found effective gene silencing sequences against FAK by screening of a plasmid library constructed for expressing shRNAs targeting FAK. The sequences of 16 clones first picked up from the library were different from those reported by other groups $(43,44)$. We also demonstrated that chemically synthesized siRNAs corresponding to shRNA target sequences inhibited FAK expression. In PC3M cancer cells, more than $90 \%$ suppression of FAK protein expression by treatment with shRNAs targeting FAK was observed within 2 days of transfection and lasted throughout the observation period. Chemically synthesized siRNAs on the basis of shRNA sequences successfully depleted up to $80 \%$ of FAK protein expression at day 3 post-transfection, indicating the same level of efficacy for siRNA as shRNA plasmid against endogenous FAK. The degree of suppression of endogenous FAK by shRNA in mouse mammary carcinoma 4T1 cells was not as strong as that shown in the human cell lines. One possible reason for this weaker effect of shRNA in mouse cells may be that the transfection efficiency was not as high in 4T1 cells compared with PC3M cells as assessed by control EGFP expression (data not shown). Another reason may be that the cell dividing speed of 4T1 cells is much higher than that of $\mathrm{PC} 3 \mathrm{M}$ cells resulting in the rapid dilution of transfected plasmids into 4T1 cells, and/or the activity of RNase in the cytoplasm which degrades shRNA may be high 

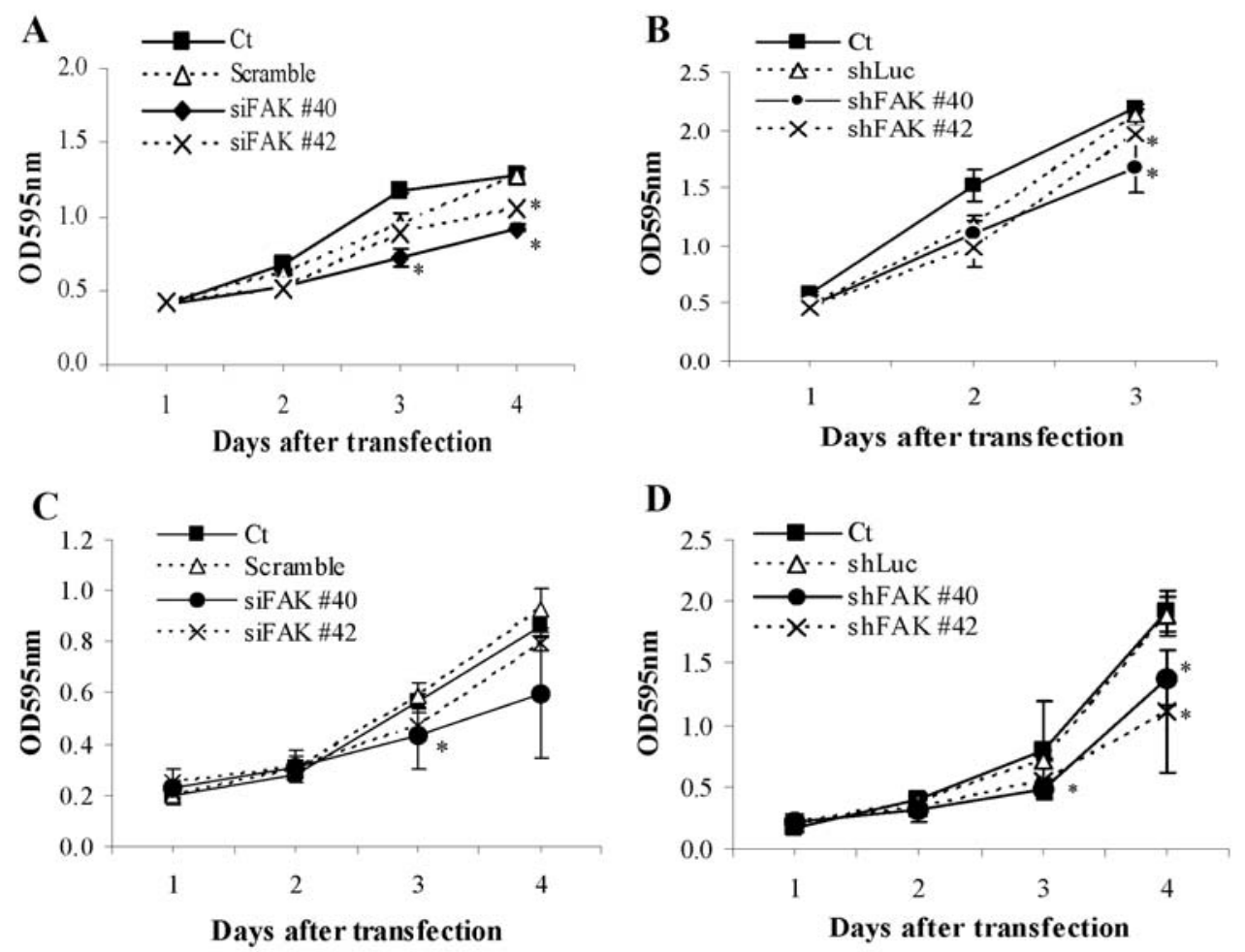

Figure 6. Inhibition of cell growth by FAK siRNA or shRNA plasmid in vitro. One day after the cell seeding on a 96-well plate, PC3M cells were transfected with FAK or scrambled siRNA (A) and 4T1 cells were transfected with FAK or luciferase shRNA plasmid (B). PC3M (C) and 4T1 (D) cells cultured on a 6-well plate after transfection with siRNA or shRNA, were seeded on a 96-well plate coated with 0.25 or $0.0625 \mu \mathrm{g} / \mathrm{well}$ laminin, respectively. Cell growth was measured daily by MTT assay. Results are expressed as means \pm SD of triplicate wells. These experiments were repeated twice with similar results. ${ }^{*} \mathrm{P}<0.05$ compared with scrambled siRNA or shLuc plasmid in (A) or (B), respectively (unpaired, two-tailed t-test with Bonferroni correction).

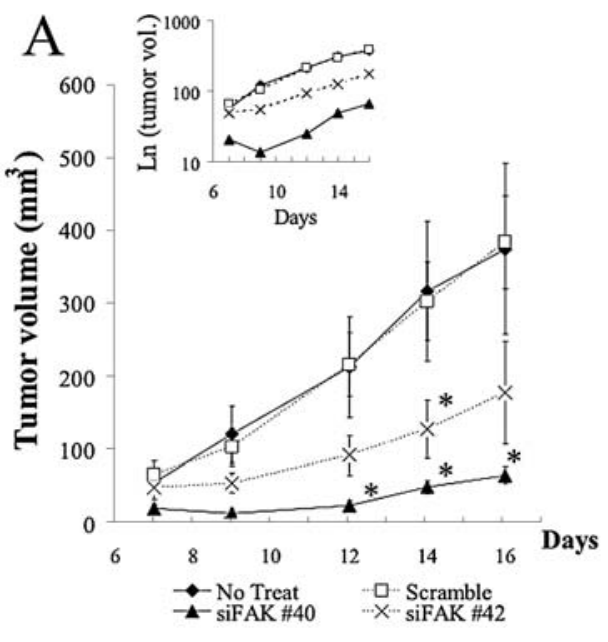

$\mathrm{C}$

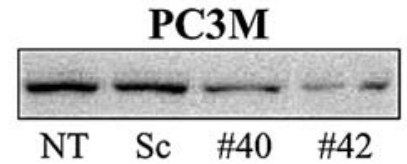

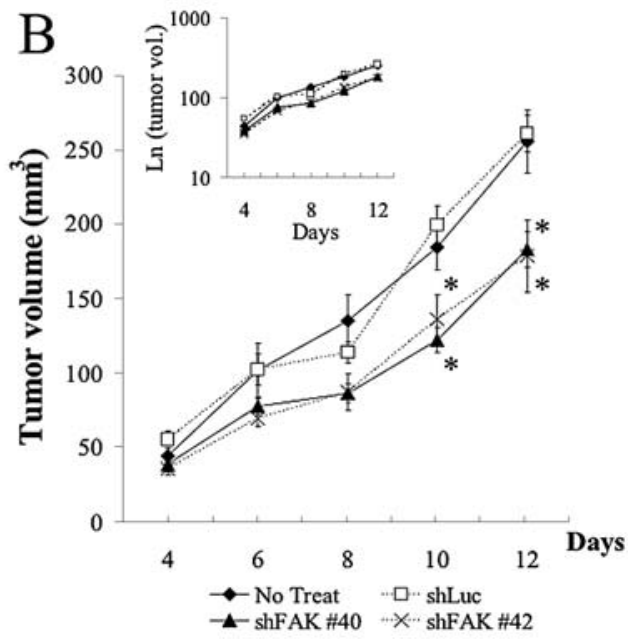

4T1

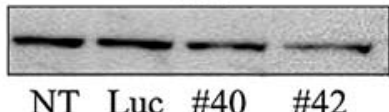

Figure 7. In vivo tumor growth inhibitory effects of FAK siRNA and shRNA plasmid. (A) PC3M cells were transfected with FAK or scrambled siRNA in $10-\mathrm{cm}$ culture dishes. One day later, tumor cells $\left(3 \times 10^{6}\right)$ were injected s.c. into the right flank of six nude mice in each group. (B) 4T1 cells were transfected with siRNA plasmid against FAK or luciferase in 10-cm culture dishes. One day later, tumor cells $\left(1 \times 10^{6}\right)$ were injected s.c. into the right mammary fat pad of six Balb/c mice in each group. Tumor growth was measured with a caliper every 2 or 3 days. The results are expressed as means \pm SE of tumor volume (mm ${ }^{3}$ ) of six mice in each group. These experiments were repeated twice with similar results. ${ }^{*} \mathrm{P}<0.05$ compared with scrambled siRNA or shLuc plasmid in (A) or (B), respectively (Mann-Whitney U test). Inset: corresponding logarithmic regression trend lines. (C) Representative of FAK expression level in the injected cells analyzed by Western blotting using anti-FAK antibody. 
in 4T1 cells. Moreover, because the plasmid library expressing shRNA was originally constructed from the cDNA of human FAK, there was one mismatch sequence at the indicated site against mouse FAK in clones \#42, \#64 and \#66, but not in clone \#40 (Fig. 2). However, we observed that clone \#42, but not \#64 or \#66, suppressed mouse FAK protein expression in 4T1 cells to the same level as that achieved by full match clone \#40. Moreover, both clones \#40 and \#42 efficiently suppressed the phosphorylation of ERK, downstream of FAK signal transduction, indicating that these clones are good tools for the further analysis of FAK function. This is consistent with studies indicating that siRNA can tolerate single mutations in the center of the molecule, and that up to four mutations are necessary for complete inactivation (45-47).

The most significant finding in recent studies is that FAK is a necessary component for the signal transduction pathways that are involved in cell migration, invasion, apoptosis, and adhesion in vitro. However, research investigating the role of FAK in cell proliferation has been limited, even in vitro. In this study, we clearly demonstrated that FAK functions are important for cell adhesion and migration, by applying synthetic siRNAs and vector-based shRNAs targeting FAK. In the cell adhesion assay, the clear inhibition of cell adhesion to fibronectin/laminin by FAK suppression was observed both in PC3M and 4T1 cells. In the haptotactic migration assay, significant inhibition of cell migration by FAK suppression was observed in PC3M cells (30 and 25\% inhibition by \#40 and \#42, respectively), and mild inhibition of cell migration was observed in 4T1 cells (7 and 18\% inhibition by $\# 40$ and \#42, respectively). These results are consistent with previous studies using antisense or siRNA oligonucleotides targeting FAK. Lowering FAK expression with antisense or siRNA oligonucleotides inhibited SW620 human colon cancer cell adhesion and the degree of FAK inhibition correlated well with the extent of inhibition of cell adhesion (43). In the A549 human adenocarcinoma cell line, FAK antisense, as well as the dominant negative FRNK, inhibited EGF-stimulated cell motility and MMP-9 secretion (41). Collectively, these results suggest that the inhibition of cell adhesion and migration by siRNA/shRNA was caused solely by FAK inhibition. It is of interest that siRNA \#42 suppress FAK protein expression (80\% inhibition) more effectively than $\# 40$ (50\% inhibition) in PC3M cells, although inhibition levels of cell adhesion and migration by each siRNA were almost the same. These results may indicate that more than $50 \%$ inhibition of FAK protein expression is enough to show the maximal effect of FAK inhibition and reach a plateau in PC3M cells.

Next, we demonstrated that the decrease of FAK expression by siRNAs/shRNAs suppressed cellular proliferation in a monolayer culture and inhibited tumor growth in both a mouse heterotopic xenograft model (human prostate cancer PC3M cells) and an orthotopic syngraft model (mouse breast cancer 4T1 cells) at the same time. The degree of suppression of in vitro tumor growth by RNAi was more potent when the cells were cultured in laminin-coated plates (36 and 21\% inhibition in PC3M cells, 34 and $41 \%$ inhibition in 4T1 cells, respectively) than in normal plates (28 and $18 \%$ inhibition in PC3M cells, 22 and $8 \%$ inhibition in $4 \mathrm{~T} 1$ cells, respectively), indicating that the effects of FAK downregulation on tumor cell growth are better reflected in an in vitro environment resembling the tumor microenvironment in the host tissue. In 4T1 cells, inhibition levels of tumor growth by shRNA targeting FAK were the same both in vitro and in vivo. Direct injection of shRNA targeting FAK into the tumor grown in mammary fat pad in the 4T1 orthotopic syngraft model also significantly reduced tumor growth (unpublished data). These results suggest that tumor growth inhibition of 4T1 cells in the in vivo model by shRNA targeting FAK, was mainly by tumor cell growth inhibition caused by FAK suppression. In PC3M cells, tumor growth in vitro was suppressed only $21-36 \%$ by siRNA compared with control, whereas tumor growth in vivo was dramatically suppressed by FAK gene silencing (58 and $84 \%$ inhibition by $\# 40$ and $\# 42$, respectively). These results may be consistent in part with a study showing that FAK siRNA did not affect human pancreatic adenocarcinoma cellular proliferation in a monolayer culture, whereas it had a tendency to attenuate tumor growth in nude mice (44), although the statistical difference was not observed. This suppression of tumor growth in vivo was especially caused by the decreased cell dividing speed observed at the early stage after the implantation of tumor cells transfected with siRNA targeting FAK. This may, of course, be caused by the suppression of tumor cell proliferation by effective siRNA, but may also be due to the suppression of cell adhesion to ECM by siRNA. In this model, s.c. injected tumor cells should first attach to ECM and then start growth upon the induction of growth signals caused by cell attachment, or otherwise lead to apoptosis. There are reports showing that attenuation of FAK expression in tumor cells results in apoptosis that is mediated by caspase 8- and FADDdependent pathways (48), and that FAK gene silencing would promote apoptosis and reverse acquired apoptosis resistance in human pancreatic adenocarcinoma cells (49). In a PC3M xenograft model, apoptosis may be accelerated by FAK suppression in the implantation site. Suppression of cell adhesion may not only reduce the growth rate but also decrease the cell number by apoptosis in the tumor implantation site, and hence decrease the tumor size.

In this study, we demonstrated for the first time, that FAK plays a very important role in tumor proliferation in vitro and in vivo, as well as in tumor migration and adhesion simultaneously. Tumor growth in vivo is a complex process in this subcutaneous injection model including cell adhesion to ECM and proliferation as discussed above. Therefore, direct injection of siRNA/shRNA into tumors that have been established in mice beforehand will address the function of FAK in tumor proliferation more clearly.

\section{References}

1. Parsons JT and Parsons SJ: Src family protein tyrosine kinases: cooperating with growth factor and adhesion signaling pathways. Curr Opin Cell Biol 9: 187-192, 1997.

2. Ilic D, Almeida EA, Schlaepfer DD, Dazin P, Aizawa S and Damsky CH: Extracellular matrix survival signals transduced by focal adhesion kinase suppress p53-mediated apoptosis. J Cell Biol 143: 547-560, 1998.

3. Zachary I and Rozengurt E: Focal adhesion kinase (p125FAK): a point of convergence in the action of neuropeptides, integrins, and oncogenes. Cell 71: 891-894, 1992.

4. Cary LA, Chang JF and Guan JL: Stimulation of cell migration by overexpression of focal adhesion kinase and its association with Src and Fyn. J Cell Sci 109: 1787-1794, 1996. 
5. Chen HC and Guan JL: Association of focal adhesion kinase with its potential substrate phosphatidylinositol 3-kinase. Proc Natl Acad Sci USA 91: 10148-10152, 1994.

6. Schaller MD: Biochemical signals and biological responses elicited by the focal adhesion kinase. Biochim Biophys Acta 1540: 1-21, 2001

7. Schaller MD and Parsons JT: pp125-FAK dependent tyrosine phosphorylation of paxillin creates a high-affinity binding site for Crk. Mol Cell Biol 15: 2635-2645, 1995.

8. Schlaepfer DD, Hanks SK, Hunter T and Van der Geer P: Integrin-mediated signal transduction linked to Ras pathway by GRB2 binding to focal adhesion kinase. Nature 372: 786-791, 1994.

9. Weiner TM, Liu ET, Craven RJ and Cance WG: Expression of growth factor receptors, the focal adhesion kinase, and other tyrosine kinases in human soft tissue tumors. Ann Surg Oncol 1: 18-27, 1994.

10. Tremblay L, Hauck W, Aprikian AG, Begin LR, Chapdelaine A and Chevalier S: Focal adhesion kinase (pp125FAK) expression, activation and association with paxillin and p50CSK in human metastatic prostate carcinoma. Int J Cancer 68: 164-171, 1996.

11. Owens LV, Xu L, Craven RJ, Dent GA, Weiner TM, Kornberg L, Liu ET and Cance WG: Overexpression of the focal adhesion kinase (p125FAK) in invasive human tumors. Cancer Res 55: 2752-2755, 1995

12. Cance WG, Harris JE, Iacocca MV, Roche E, Yang X, Chang J, Simkins S and Xu L: Immunohistochemical analyses of focal adhesion kinase expression in benign and malignant human breast and colon tissues: correlation with preinvasive and invasive phenotypes. Clin Cancer Res 6: 2417-2423, 2000.

13. Schlaepfer DD, Hauck CR and Sieg DJ: Signaling through focal adhesion kinase. Prog Biophys Mol Biol 71: 435-478, 1999.

14. Parsons JT, Martin KH, Slack JK, Taylor JM and Weed SA: Focal adhesion kinase: a regulator of focal adhesion dynamics and cell movement. Oncogene 19: 5606-5613, 2000.

15. Hauck CR, Hsia DA, Puente XS, Cheresh DA and Schlaepfer DD: FRNK blocks v-Src-stimulated invasion and experimental metastases without effects on cell motility or growth. EMBO J 21: 6289-6302, 2002.

16. Hecker TP and Gladson CL: Focal adhesion kinase in cancer. Front Biosci 8: s705-s714, 2003.

17. Hungerford JE, Compton MT, Matter ML, Hoffstrom BG and Otey CA: Inhibition of pp $125 \mathrm{FAK}$ in cultured fibroblasts results in apoptosis. J Cell Biol 135: 1383-1390, 1996.

18. Frisch SM, Vuori K, Ruoslahti E and Chan-Hui PY: Control of adhesion-dependent cell survival by focal adhesion kinase. J Cell Biol 134: 793-799, 1996.

19. Zhao JH, Reiske H and Guan JL: Regulation of the cell cycle by focal adhesion kinase. J Cell Biol 143: 1997-2008, 1998.

20. Leyton J, Garcia-Marin LJ, Tapia JA, Jensen RT and Moody TW: Bombesin and gastrin releasing peptide increase tyrosine phosphorylation of focal adhesion kinase and paxillin in nonsmall cell lung cancer cells. Cancer Lett 162: 87-95, 2001.

21. Yamamoto D, Sonoda Y, Hasegawa M, Funakoshi-Tago M, Aizu-Yokota E and Kasahara T: FAK overexpression upregulates cyclin D3 and enhances cell proliferation via the PKC and PI3kinase-Akt pathways. Cell Signal 15: 575-583, 2003.

22. Taylor JM, Mack CP, Nolan K, Regan CP, Owens GK and Parsons JT: Selective expression of an endogenous inhibitor of FAK regulates proliferation and migration of vascular smooth muscle cells. Mol Cell Biol 21: 1565-1572, 2001.

23. Richardson A and Parsons JT: A mechanism for regulation of the adhesion-associated protein tyrosine kinase pp125FAK. Nature 380: 538-540, 1996.

24. Taylor JM, Rovin JD and Parsons JT: A role for focal adhesion kinase in phenylephrine-induced hypertrophy of rat ventricular cardiomyocytes. J Biol Chem 275: 19250-19257, 2000.

25. Schaller MD, Borgman CA and Parsons JT: Autonomous expression of a noncatalytic domain of the focal adhesionassociated protein tyrosine kinase pp125FAK. Mol Cell Biol 13: 785-791, 1993.

26. Bertrand JR, Pottier M, Vekris A, Opolon P, Maksimenko A and Malvy C: Comparison of antisense oligonucleotides and siRNAs in cell culture and in vivo. Biochem Biophys Res Commun 296: 1000-1004, 2002.
27. Aoki Y, Cioca DP, Oidaira H, Kamiya J and Kiyosawa K: RNA interference may be more potent than antisense RNA in human cancer cell lines. Clin Exp Pharmacol Physiol 30: 96-102, 2003.

28. Sharp PA: RNA interference-2001. Genes Dev 15: 485-490, 2001.

29. Hannon GJ: RNA interference. Nature 418: 244-251, 2002.

30. Elbashir SM, Harborth J, Lendeckel W, Yalcin A, Weber K and Tuschl T: Duplexes of 21-nucleotide RNAs mediate RNA interference in cultured mammalian cells. Nature 411: 494-498, 2001.

31. McCaffrey AP, Meuse L, Pham TT, Conklin DS, Hannon GJ and Key MA: RNA interference in adult mice. Nature 418: 38-39, 2002.

32. Tiscornia G, Singer O, Ikawa M and Verma IM: A general method for gene knockdown in mice by using lentiviral vectors expressing small interfering RNA. Proc Natl Acad Sci USA 100: 1844-1848, 2003.

33. Carmell MA, Zhang L, Conklin DS, Hannon GJ and Rosenquist TA: Germline transmission of RNAi in mice. Nat Struct Biol 10: 91-92, 2003.

34. Brummelkamp TR, Bernards R and Agami R: A system for stable expression of short interfering RNAs in mammalian cells. Science 296: 550-553, 2002.

35. Paddison PJ, Caudy AA, Bernstein E, Hannon GJ and Conklin DS: Short hairpin RNAs (shRNAs) induce sequence-specific silencing in mammalian cells. Genes Dev 16: 948-958, 2002.

36. Sui G, Soohoo C, Affar EB, Gay F, Shi Y, Forrester WC and Shi Y: A DNA vector-based RNAi technology to suppress gene expression in mammalian cells. Proc Natl Acad Sci USA 99: 5515-5520, 2002

37. Yu JY, DeRuiter SL and Turner DL: RNA interference by expression of short-interfering RNAs and hairpin RNAs in mammalian cells. Proc Natl Acad Sci USA 99: 6047-6052, 2002.

38. Miyagishi $M$ and Taira K: U6 promoter-driven siRNAs with four uridine 3' overhangs efficiently suppress targeted gene expression in mammalian cells. Nat Biotechnol 20: 497-500, 2002.

39. Lee NS, Dohjima T, Bauer G, Li H, Li M-J, Ehsani A, Salvaterra P and Rossi J: Expression of small interfering RNAs targeted against HIV-1 rev transcripts in human cells. Nat Biotechnol 20: 500-505, 2002.

40. Paul CP, Good PD, Winer I and Engelke DR: Effective expression of small interfering RNA in human cells. Nat Biotechnol 20: 505-508, 2002.

41. Hauck CR, Sieg DJ, Hsia DA, Loftus JC, Gaarde WA, Monia BP and Schlaepfer DD: Inhibition of focal adhesion kinase expression or activity disrupts epidermal growth factorstimulated signaling promoting the migration of invasive human carcinoma cells. Cancer Res 61: 7079-7090, 2001.

42. Braasch DA and Corey DR: Novel antisense and peptide nucleic acid strategies for controlling gene expression. Biochemistry 41: 4503-4510, 2002

43. Walsh MF, Thamilselvan V, Grotelueschen R, Farhana L and Basson MD: Absence of adhesion triggers differential FAK and SAPKp38 signals in SW620 human colon cancer cells that may inhibit adhesiveness and lead to cell death. Cell Physiol Biochem 13: 135-146, 2003.

44. Duxbury MS, Ito H, Benoit E, Zinner MJ, Ashley SW and Whang EE: RNA interference targeting focal adhesion kinase enhances pancreatic adenocarcinoma gemcitabine chemosensitivity. Biochem Biophys Res Commun 311: 786-792, 2003.

45. Holen T, Amarzguioui M, Wiiger MT, Babaie E and Prydz H: Positional effects of short interfering RNAs targeting the human coagulation trigger Tissue Factor. Nucleic Acids Res 30: 1757-1766, 2002

46. Jacque JM, Triques $\mathrm{K}$ and Stevenson M: Modulation of HIV-1 replication by RNA interference. Nature 418: 435-438, 2002.

47. Leirdal $M$ and Sioud M: Gene silencing in mammalian cells by preformed small RNA duplexes. Biochem Biophys Res Commun 295: 744-748, 2002.

48. Kurenova E, Xu LH, Yang X, Baldwin Jr AS, Craven RJ, Hanks SK, Liu Z and Cance WG: Focal adhesion kinase suppresses apoptosis by binding to the death domain of receptor-interacting protein. Mol Cell Biol 24: 4361-4371, 2004

49. Duxbury MS, Ito H, Zinner MJ, Ashley SW and Whang EE: Focal adhesion kinase gene silencing promotes anoikis and suppresses metastasis of human pancreatic adenocarcinoma cells. Surgery 135: 555-562, 2004. 